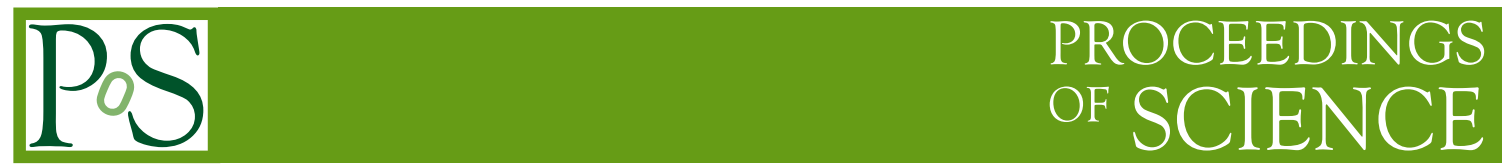

\title{
Nuclear forces: Theory and applications
}

\section{E. Epelbaum}

Institut für Theoretische Physik II, Fakultät für Physik und Astronomie, Ruhr-Universität Bochum 44780 Bochum, Germany

E-mail: evgeny . epelbaum@rub. de

I review the status of nuclear chiral effective field theory and discuss selected applications focusing on current topics such as the structure of three-nucleon force. I also discuss the discretized version of this approach which treats pions and nucleons as point-like particles on an Euclidean space-time lattice. This allows one to evaluate the path integral by Monte Carlo sampling and to access the properties of heavier systems.

Sixth International Conference on Quarks and Nuclear Physics,

April 16-20, 2012

Ecole Polytechnique, Palaiseau, Paris 


\section{Introduction}

Understanding the properties of atomic nuclei and nuclear dynamics from first principles remains to be a major challenge. Complementary to first attempts along these lines based on lattice QCD, see e.g. [1], an effective field theory (EFT) approach has been extensively used in the last two decades to describe the properties of nuclear bound states and reactions $[2,3,4,5]$. This method exploits the separation of scales exhibited in nuclear systems and is based on the effective chiral Lagrangian formulated in terms of pions and nucleons (and sometimes also the $\Delta(1232)$ isobars) which are the proper degrees of freedom (DOF) for this kind of problems. As pions are the pseudoGoldstone bosons of the approximate chiral symmetry of QCD, their interaction are of derivative nature. This allows one to describe low-energy pion dynamics in chiral perturbation theory by employing a simultaneous expansion in external momenta and about the chiral limit.

While this approach can be straightforwardly extended to the single-baryon sector, its application to nuclear dynamics requires modifications in order to account for nonperturbative features of the nuclear force. Thanks to the nonrelativistic nature of the problem at hand, the description of low-energy nuclear dynamics simplifies enormously and can be formulated in terms of the conventional, quantum mechanical $A$-body problem. Combining this formulation with chiral EFT allows one to derive nuclear forces and exchange currents in harmony with the symmetries of QCD in a model-independent and systematically improvable way. In this talk I outline some recent developments along these lines. The paper is organized as follows. In section 2 I describe the status of the nuclear forces in chiral EFT and discuss some actual topics focusing especially on the threenucleon force. I then switch in section 3 to a discretized version of chiral EFT which allows one to use stochastic methods in order to access the properties of nuclei and present selected recent results for up to $A=12$ nucleons. Finally, a brief summary and outlook are given in section 4 .

\section{Towards a precision determination of the nuclear force in chiral EFT}

The usefulness of an EFT framework relies on the separation of scales. For the case at hand, the soft scale $Q$ is associated with the typical external momenta of the nucleons which are assumed to be of the order of the pion mass while the hard scale $\Lambda_{\chi}$ can be estimated e.g. by the mass of the $\rho$-meson. The long-range part of the nuclear force and current operators is, of course, governed by soft physics mediated by exchange of pions. Long-range interactions can be worked out systematically, order-by-order in the chiral expansion and are strongly constrained by the chiral symmetry of QCD. Short-range forces, on the other hand, are driven by physics that cannot be resolved in low-energy reactions. They can be mimicked by zero-range contact interactions with an increasing number of derivatives. The chiral symmetry of QCD does not provide any constraints on the contact interactions (except for their quark mass dependence).

Presently, the most advanced calculations of the two-nucleon force (2NF) are carried out at next-to-next-to-next-to-leading order $\left(\mathrm{N}^{3} \mathrm{LO}\right)$ using the heavy-baryon formulation of the chiral effective Lagrangian for pions and nucleons $[6,7]$. At this order, the long-range part of the nuclear force receives contributions from exchange of up to three pions. Fig. 3 illustrates the chiral expansion for the two most important components of the 2NF, namely for the isovector-tensor and isoscalar-central potentials. All low-energy constants (LECs) appearing in the expressions for the 

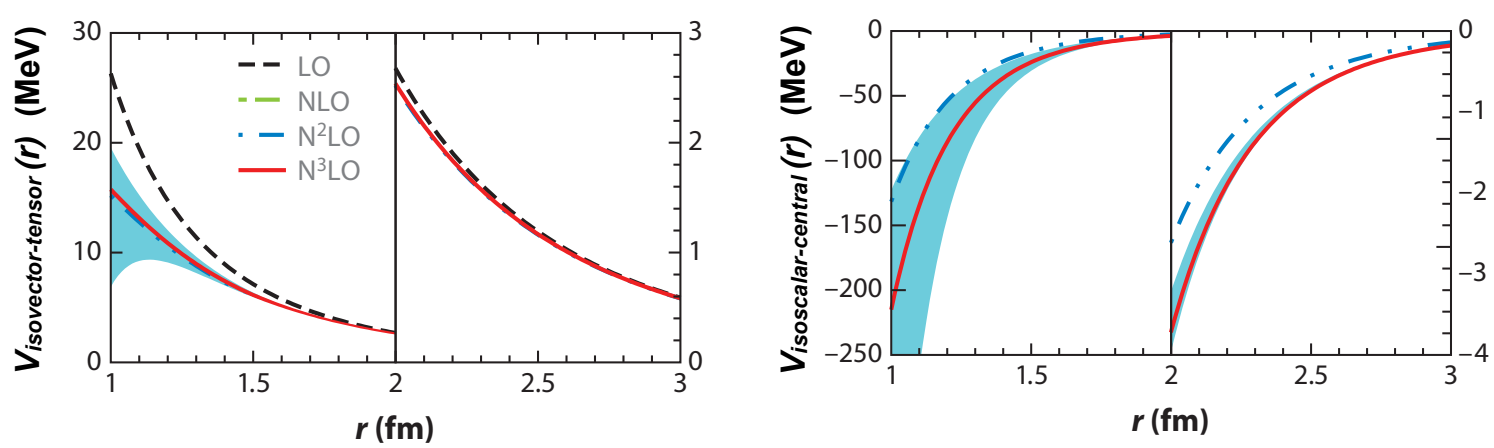

Figure 1: Chiral expansion of the isovector-tensor (left panel) and isoscalar central (right panel) long-range potentials. The shaded band shows the estimated size of the short-range contributions represented by the (smeared) contact interactions, see Ref. [5] for more details.

long-range part of $2 \mathrm{NF}$ are determined from pion-nucleon scattering. The potential in the isovectortensor channel is, of course, dominated by the one-pion exchange. Two-pion exchange contributions in this channel become visible at distances of the order $r \sim 2 \mathrm{fm}$ and smaller. The strong, attractive isoscalar-central potential of intermediate range is another well-known feature of the two-nucleon force. Phenomenologically, it is attributed to the correlated two-pion exchange which is often modeled in terms of the $\sigma$-meson. In chiral EFT, all low-energy manifestations of the $\sigma$ and other heavy mesons are systematically taken into account through values of the LECs in the effective Lagrangian. The strong attraction resulting from the chiral two-pion exchange potential in the isoscalar-central channel is in agreement with the phenomenological picture.

The short-range part of the $\mathrm{N}^{3} \mathrm{LO}$ potential receives contributions from 24 isospin-conserving and 2 isospin-breaking contact interactions. ${ }^{1}$ Using the appropriately chosen, finite cutoff to regularize the Schrödinger equation along the lines of Refs. [13, 15], the LECs accompanying these short-range operators have been determined from fits to Nijmegen partial wave analysis [6] and also directly to the available experimental data on nucleon-nucleon scattering [7]. The resulting $\mathrm{N}^{3} \mathrm{LO}$ potentials allow for an accurate description of nucleon-nucleon scattering observables up to energies of the order $E_{\text {lab }} \sim 200 \mathrm{MeV}$, see Fig. 2 for two representative examples, and provide a solid basis for few- and many-nucleon calculations.

Three-nucleon forces (3NF) represent an old but still very important topic in nuclear physics. While effects of the 3NF in low-energy nuclear observables are expected to be fairly small [2], they appear to be important at the level of accuracy which nowadays represents a standard in ab-initio few-body [21] as well as many-body calculations, see e.g. Ref. [22]. In spite of many decades of effort, the detailed structure of the $3 \mathrm{NF}$ is not properly described by the phenomenological models [21]. Given the very rich spin-momentum structure of the $3 \mathrm{NF}$ as compared to the $2 \mathrm{NF}$, scarcer database and relatively high computational cost, further progress in this fields clearly requires input from theory. This provides a strong motivation to study the structure of the 3NF within chiral EFT.

The first contribution to the $3 \mathrm{NF}$ appears at $\mathrm{N}^{2} \mathrm{LO}[23,24]$ and depends on two LECs that

\footnotetext{
${ }^{1}$ These numbers are based on naive dimensional analysis which underlies the chiral power counting. There is no consensus in the community regarding the validity of the naive dimensional analysis in the context of nuclear EFT, see Refs. $[13,14,15,16,17,18,19,20]$ for samples of different views on this issue.
} 

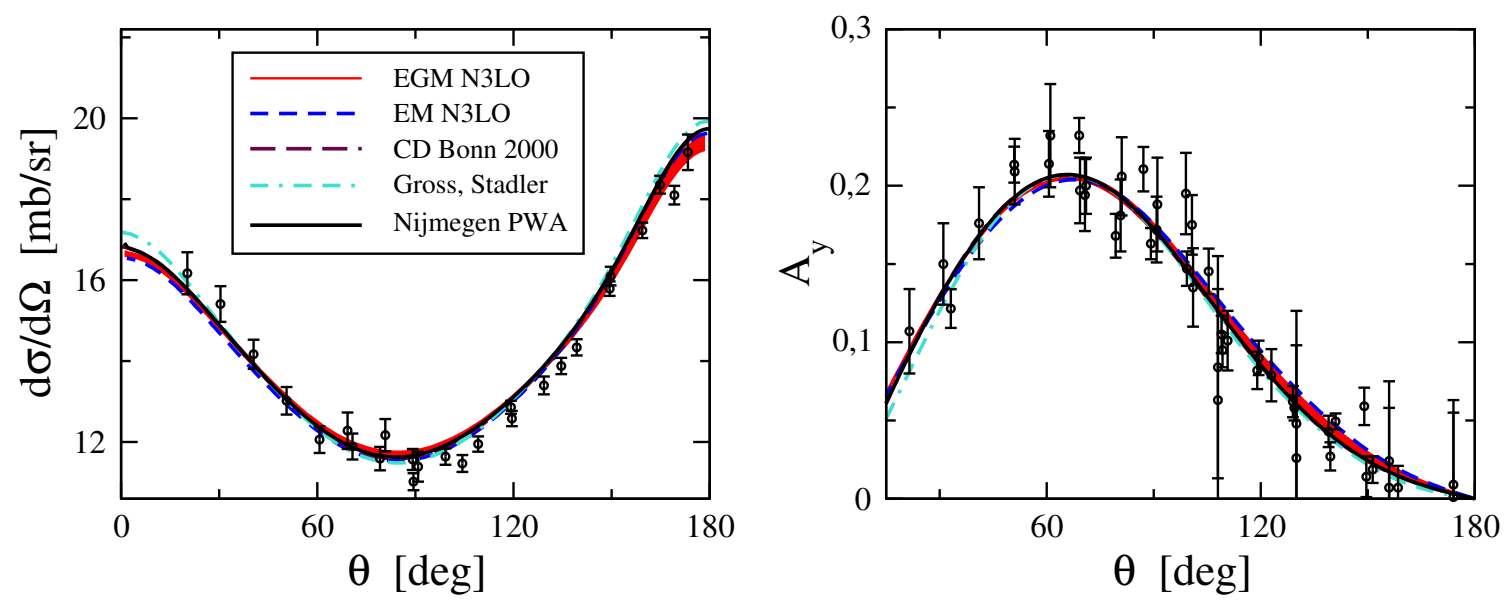

Figure 2: Neutron-proton differential cross section (left panel) and analyzing power (right panel) at $E_{\text {lab }}=50$ $\mathrm{MeV}$ calculated using chiral EFT, the CD Bonn 2000 potential of Ref. [8] and the potential developed by Gross and Stadler in Ref. [9]. Also shown are results from the Nijmegen partial wave analysis [10]. References to data can be found in [10].

have to be fixed from few-nucleon data such as e.g. the ${ }^{3} \mathrm{H}$ binding energy and the $n d$ spin-dublet scattering length. The resulting nuclear Hamiltonian at order $\mathrm{N}^{2} \mathrm{LO}$ has been applied to compute various $3 \mathrm{~N}$ scattering observables, see $[3,5,21]$ and references therein. Apart from few exceptions, one observes a good description of experimental data at low energy. However, at energies $E_{\text {lab }} \sim 50$ $\mathrm{MeV}$ and higher, the theoretical uncertainty becomes rather large. The chiral $3 \mathrm{NF}$ at $\mathrm{N}^{2} \mathrm{LO}$ is also being extensively studied in connection with the spectra of light and medium-mass nuclei and the properties of nuclear matter, see Refs. [22, 25, 26] for some recent applications.

The corrections to the $3 \mathrm{NF}$ at $\mathrm{N}^{3} \mathrm{LO}$ are worked out in Refs. [27, 28, 29] and do not involve any unknown LECs. At this order and, in fact, also at next-to-next-to-next-to-next-to-leading order $\left(\mathrm{N}^{4} \mathrm{LO}\right)$, the long-range part of the $3 \mathrm{NF}$ is given by three topologies. The longest-range contribution clearly emerges from two-pion exchange (TPE) graphs, in which a single pion is exchanged between the two nucleon pairs. The next-longest-range terms are given by diagrams with one- and two-pion exchange between the two nucleon pairs (OPE-TPE) and by the so-called ring graphs, in which a virtual pion scatters once off every one of the three nucleons.

The dominant longest-range $3 \mathrm{NF}$ mechanism is usually associated with the intermediate $\Delta(1232)$ excitation. It is taken into account in all models of the $3 \mathrm{NF}$ and also drives the leading contribution to the chiral TPE $3 \mathrm{NF}$ at $\mathrm{N}^{2} \mathrm{LO}$. More precisely, in the formulation of chiral EFT based on pions and nucleons as the only explicit DOF, all effects of the $\Delta$-isobar (and higher excitations of the nucleon) are encoded in the values LECs of the effective Lagrangian. The subleading pion-nucleon LECs $c_{3,4}$, which determine the strength of the TPE $3 \mathrm{NF}$ at $\mathrm{N}^{2} \mathrm{LO}$, are, in fact, to a large extent saturated by the $\Delta$-isobar. The situation is different for the OPE-TPE and ring topologies whose (nominally) leading contributions emerge at $\mathrm{N}^{3} \mathrm{LO}$ and are driven by the lowest-order $\pi N$ vertices which do not incorporate the physics of the $\Delta$-isobar. One, therefore, expects that the $\mathrm{N}^{3} \mathrm{LO}$ results for the OPE-TPE and ring $3 \mathrm{NF}$ are not yet converged. As a consequence, it is needed to either go to higher orders in the chiral expansion beyond $\mathrm{N}^{3} \mathrm{LO}$ or to calculate explicitly the contributions of the $\Delta$-isobar at $\mathrm{N}^{3} \mathrm{LO}$ using an extended chiral EFT formulation with explicit Delta DOF, see also 

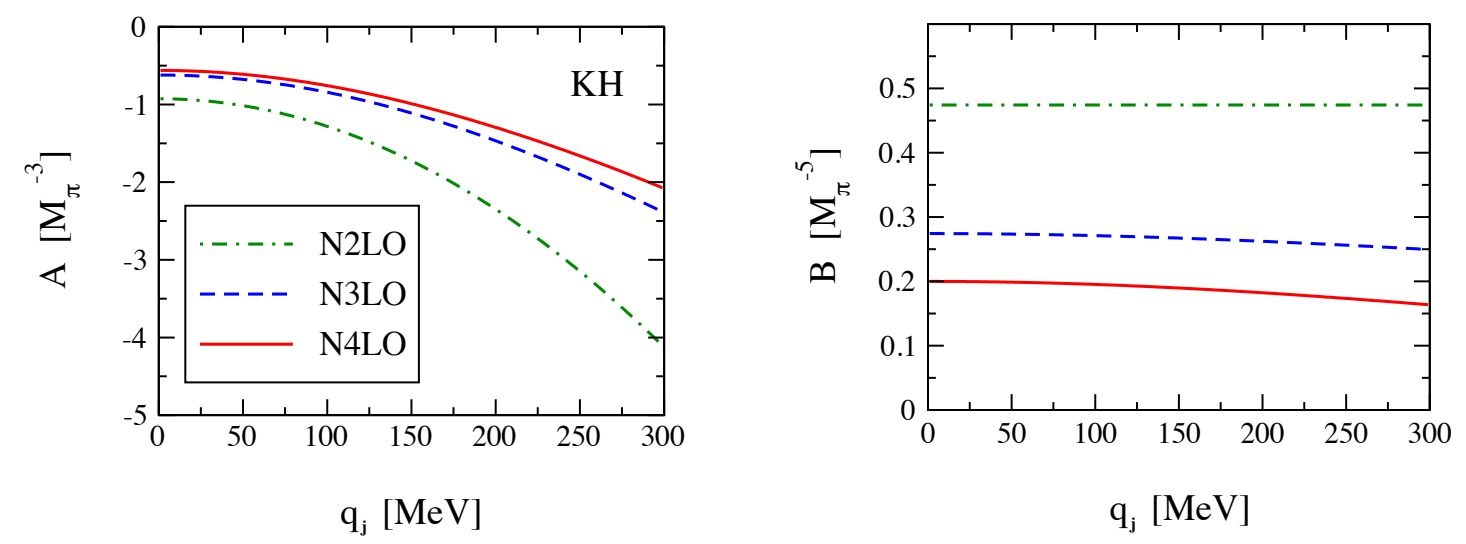

Figure 3: Chiral expansion of the functions $\mathscr{A}\left(q_{j}\right)$ and $\mathscr{B}\left(q_{j}\right)$ entering the TPE $3 \mathrm{NF}$ up to $\mathrm{N}^{4} \mathrm{LO}$. The results are obtained with the LECs determined from the order- $Q^{4}$ fit to the Karlsruhe-Helsinki partial wave analysis of pion-nucleon scattering of Ref. [11].

the discussion in Ref. [30]. The first step along these lines was made recently in Ref. [31], where the chiral expansion of the longest-range, TPE $3 \mathrm{NF}$ topology was extended to $\mathrm{N}^{4} \mathrm{LO}$ within the $\Delta$-less theory. In the isospin and static limits, the general structure of the TPE $3 \mathrm{NF}$ in momentum space has the form

$$
V_{2 \pi}=\frac{\vec{\sigma}_{i} \cdot \vec{q}_{i} \vec{\sigma}_{k} \cdot \vec{q}_{k}}{\left[q_{i}^{2}+M_{\pi}^{2}\right]\left[q_{k}^{2}+M_{\pi}^{2}\right]}\left(\tau_{i} \cdot \tau_{k} \mathscr{A}\left(q_{j}\right)+\tau_{i} \times \tau_{k} \cdot \tau_{j} \vec{q}_{i} \times \vec{q}_{k} \cdot \vec{\sigma}_{j} \mathscr{B}\left(q_{j}\right)\right),
$$

where $\vec{\sigma}_{i}$ denote the Pauli spin matrices for the nucleon $i, \vec{q}_{i}=\vec{p}_{i}{ }^{\prime}-\vec{p}_{i}$, with $\vec{p}_{i}{ }^{\prime}$ and $\vec{p}_{i}$ being the final and initial momenta of the nucleon $i$ and $q_{i} \equiv\left|\vec{q}_{i}\right|$. The quantities $\mathscr{A}\left(q_{j}\right)$ and $\mathscr{B}\left(q_{j}\right)$ in Eq. (2.1) are scalar functions of the momentum transfer of the $j$-th nucleon whose explicite form is derived within the chiral expansion. The $\mathrm{N}^{4} \mathrm{LO}$ contributions to $\mathscr{A}$ and $\mathscr{B}$ worked out in Ref. [31] depend on certain combinations of LECs from the order- $Q^{2}, Q^{3}$ and $Q^{4}$ effective $\pi N$ Lagrangian, whose values were extracted from pion-nucleon scattering. As shown in Fig. 3, one observes a good convergence of the chiral expansion for the functions $\mathscr{A}$ and $\mathscr{B}$ which is fully in line with the expectations based on the qualitative arguments given above. The extension of the $\mathrm{N}^{4} \mathrm{LO}$ calculation to the more interesting case of the OPE-TPE and ring topologies is in progress. The contact interactions at $\mathrm{N}^{4} \mathrm{LO}$ are considered in Ref. [32]. We further emphasize that the numerical implementation of the novel $3 \mathrm{NF}$ terms requires carrying out of a partial wave decomposition. This can be carried out numerically using the technique developed in Ref. [33].

\section{Nuclear lattice simulations}

Recently, a discretized version of chiral EFT has been proposed and successively used to compute the properties of few- and many-nucleon systems. In this framework, pions and nucleons are treated as point-like particles on an Euclidean space-time lattice, and the path integral is evaluated by Monte Carlo sampling [34, 35, 36, 37]. Using hadronic DOF rather than quarks and gluons allows one to probe large volumes and greater numbers of nucleons as compared to lattice QCD. Clearly, the method is only applicable at low energies where chiral EFT is expected to converge. 
The crucial object to calculate in our nuclear lattice simulations is the correlation function for $A$ nucleons in the Euclidean space-time

$$
Z_{A}(t)=\left\langle\Psi_{A}|\exp (-t H)| \Psi_{A}\right\rangle .
$$

Here, the states $\left|\Psi_{A}\right\rangle$ refer to the Slater determinants for $A$ free nucleons, $H$ is the Hamiltonian of the system and $t$ the Euclidean time. The correlation function can be most conveniently calculated utilizing the Hubbard-Stratonovich transformation in order to get rid of interaction terms quartic in the nucleon fields (at the expense of introducing interactions with auxiliary fields) and employing hybrid Monte Carlo technique, see [37] for more details. Once $Z_{A}(t)$ is computed, the ground state energy of the $A$-nucleon system can be extracted from the asymptotic behavior of the correlation function for large $t$,

$$
E_{A}^{0}=-\lim _{t \rightarrow \infty} \frac{d}{d t} \ln Z_{A}(t)
$$

Similarly, one can obtain expectation value of a normal ordered operator $\mathscr{O}$ via

$$
\left\langle\Psi_{A}^{0}|\mathscr{O}| \Psi_{A}^{0}\right\rangle=\lim _{t \leftarrow \infty} \frac{Z_{A}^{\mathscr{O}}(t)}{Z_{A}(t)}, \quad Z_{A}^{\mathscr{O}}(t)=\left\langle\Psi_{A}|\exp (-t H / 2) \mathscr{O} \exp (-t H / 2)| \Psi_{A}\right\rangle .
$$

In order to define the action, one first needs to tune two-nucleon contact operators to reproduce NN scattering data. Thus, one needs to compute two-particle phase shifts on the lattice. Instead of using Lüscher's formula [38], we employ here the spherical wall method which allows us to determine phase shifts and mixing angles from measuring the energy spectrum for two particles with specifically chosen boundary conditions, see [39] for more details. Last but not least, it should be emphasized that we use the lowest-order action in our simulations which incorporates the physics of the one-pion exchange and the leading contact interactions. Higher-order contributions and $3 \mathrm{NFs}$ are taken into account perturbatively.

The most advanced studies within this framework are so far carried out at $\mathrm{N}^{2} \mathrm{LO}$ in the chiral expansion [40, 41, 42, 43]. We take into account the appropriate isospin-breaking corrections and Coulomb interaction, see [42] for more details, and the contributions of the leading 3NF. The two LEC entering the $3 \mathrm{NF}$ are fixed in Ref. [43] from the triton and $\alpha$-particle binding energies. With the nuclear Hamiltonian being fixed as described above, we found $-58(2) \mathrm{MeV}$ and -91 (3) $\mathrm{MeV}$ for the ground state energies of ${ }^{8} \mathrm{Be}$ and ${ }^{12} \mathrm{C} .{ }^{2}$ These results are in a very good agreement with the corresponding experimental values of $-56.50 \mathrm{MeV}$ and $-92.16 \mathrm{MeV}$, respectively. For ${ }^{12} \mathrm{C}$, we also extracted the energies of the two lowest exciting states by generalizing the Euclidean time projection method to a multi-channel calculation. Our results are summarized in Fig. 4. All calculated energies are in agreement with experimental values. While the ground and spin- 2 states of ${ }^{12} \mathrm{C}$ have been also calculated by other groups using different methods, our results for the second spin-0 state, the famous Hoyle state, are the first ab initio calculations.

\section{Summary and outlook}

In this talk I outlined some recent developments in low-energy few- and many-nucleon chiral dynamics. One of the most interesting topics is presently the three-nucleon force. The leading $3 \mathrm{NF}$

\footnotetext{
${ }^{2}$ Here and in what follows, the error bars are one standard deviation estimates which include both Monte Carlo statistical errors and uncertainties due to extrapolation at large Euclidean time.
} 


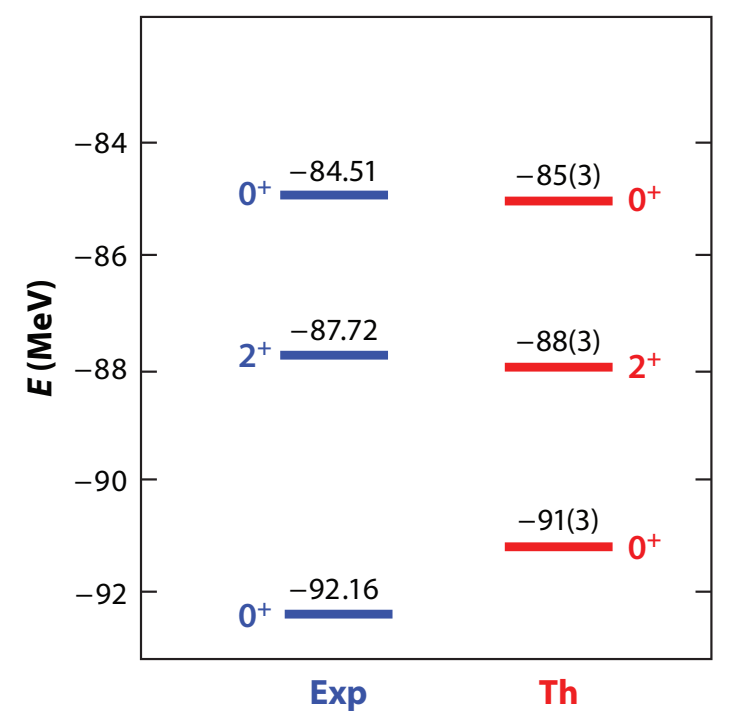

Figure 4: The ground state energy and the first excited states of ${ }^{12} C$ obtained from the nuclear lattice simulations at $\mathrm{N}^{2} \mathrm{LO}$ in comparison with experiment.

contributions at $\mathrm{N}^{2} \mathrm{LO}$ have already been extensively explored in few- and many-body calculations. There is strong evidence that the recently derived corrections to the $3 \mathrm{NF}$ at $\mathrm{N}^{3} \mathrm{LO}$ are still insufficient for an accurate description of the long-range potentials in certain channels so that one needs to go to even higher order and/or include the $\Delta$ isobar as an explicit degree of freedom. Work along these lines is in progress.

I also discussed a discretized version of chiral EFT which merges the advantages of chiral EFT with Monte Carlo techniques to access the properties of light nuclei and nuclear matter. We applied this method to study the spectra of nuclei with up to $A=12$ nucleons at $\mathrm{N}^{2} \mathrm{LO}$ in the chiral expansion and succeeded, for the first time, to describe the famous Hoyle state $a b$ initio.

It is a pleasure to thank the organizers of QNP2012 for making this exciting conference possible and all my collaborators for sharing their insights into the discussed topics. This work was supported by the European Research Council (ERC-2010-StG 259218 NuclearEFT).

\section{References}

[1] S. R. Beane ei al., arXiv:1206.5219 [hep-lat].

[2] S. Weinberg, Phys. Lett. B 251, 288 (1990).

[3] E. Epelbaum, H. -W. Hammer and U. -G. Meißner, Rev. Mod. Phys. 81, 1773 (2009).

[4] R. Machleidt and D. R. Entem, Phys. Rept. 503, 1 (2011) [arXiv:1105.2919 [nucl-th]].

[5] E. Epelbaum and U. -G. Meißner, arXiv:1201.2136 [nucl-th], to appear in Ann. Rev. Nucl. Part. Sci..

[6] E. Epelbaum, W. Glockle and U. -G. Meißner, Nucl. Phys. A 747, 362 (2005) [nucl-th/0405048].

[7] D. R. Entem and R. Machleidt, Phys. Rev. C 68, 041001 (2003) [nucl-th/0304018].

[8] R. Machleidt, Phys. Rev. C 63, 024001 (2001) [nucl-th/0006014]. 
[9] F. Gross and A. Stadler, Phys. Rev. C 78, 014005 (2008) [arXiv:0802.1552 [nucl-th]].

[10] NN-Online, see http://nn-online.org.

[11] R. Koch, Nucl. Phys. A 448, 707 (1986).

[12] R. A. Arndt, W. J. Briscoe, I. I. Strakovsky and R. L. Workman, Phys. Rev. C 74, 045205 (2006).

[13] G. P. Lepage, "How to renormalize the Schrodinger equation," [nucl-th/9706029].

[14] M. Pavon Valderrama and E. Ruiz Arriola, Phys. Rev. C 72, 054002 (2005) [nucl-th/0504067].

[15] E. Epelbaum, J. Gegelia, Eur. Phys. J. A41 (2009) 341.

[16] A. Nogga, R. G. E. Timmermans and U. van Kolck, Phys. Rev. C 72, 054006 (2005).

[17] E. Epelbaum and U. -G. Meißner, nucl-th/0609037.

[18] B. Long and U. van Kolck, Annals Phys. 323, 1304 (2008) [arXiv:0707.4325 [quant-ph]].

[19] C. -J. Yang, C. .Elster and D. R. Phillips, Phys. Rev. C 80, 044002 (2009) [arXiv:0905.4943 [nucl-th]].

[20] M. C. Birse, [arXiv:1012.4914 [nucl-th]].

[21] N. Kalantar-Nayestanaki, E. Epelbaum, J. G. Messchendorp, A. Nogga, [arXiv:1108.1227 [nucl-th]].

[22] R. Roth, J. Langhammer, A. Calci, S. Binder, P. Navratil, Phys. Rev. Lett. 107 (2011) 072501.

[23] U. van Kolck, Phys. Rev. C 49, 2932 (1994).

[24] E. Epelbaum et al., Phys. Rev. C 66, 064001 (2002) [nucl-th/0208023].

[25] K. Hebeler, J. M. Lattimer, C. J. Pethick, A. Schwenk, Phys. Rev. Lett. 105 (2010) 161102.

[26] T. Otsuka, T. Suzuki, J. D. Holt, A. Schwenk, Y. Akaishi, Phys. Rev. Lett. 105 (2010) 032501.

[27] S. Ishikawa, M. R. Robilotta, Phys. Rev. C76 (2007) 014006.

[28] V. Bernard, E. Epelbaum, H. Krebs, U.-G. Meißner, Phys. Rev. C77 (2008) 064004.

[29] V. Bernard, E. Epelbaum, H. Krebs, U.-G. Meißner, Phys. Rev. C84 (2011) 054001.

[30] R. Machleidt, D. R. Entem, J. Phys. G G37 (2010) 064041.

[31] H. Krebs, A. Gasparyan and E. Epelbaum, arXiv:1203.0067 [nucl-th], to appear in Phys. Rev. C.

[32] L. Girlanda, A. Kievsky and M. Viviani, Phys. Rev. C 84, 014001 (2011) [arXiv:1102.4799 [nucl-th]].

[33] J. Golak et al., Eur. Phys. J. A 43 (2010) 241 arXiv:0911.4173 [nucl-th].

[34] H. M. Muller, S. E. Koonin, R. Seki, U. van Kolck, Phys. Rev. C61 (2000) 044320.

[35] D. Lee, B. Borasoy, T. Schafer, Phys. Rev. C70 (2004) 014007.

[36] B. Borasoy, E. Epelbaum, H. Krebs, D. Lee, U.-G. Meißner, Eur. Phys. J. A31 (2007) 105.

[37] D. Lee, Prog. Part. Nucl. Phys. 63 (2009) 117.

[38] M. Luscher, Commun. Math. Phys. 105 (1986) 153.

[39] B. Borasoy, E. Epelbaum, H. Krebs, D. Lee, U.-G. Meißner, Eur. Phys. J. A34 (2007) 185.

[40] E. Epelbaum, H. Krebs, D. Lee, U.-G. Meißner, Eur. Phys. J. A41 (2009) 125.

[41] E. Epelbaum, H. Krebs, D. Lee, U.-G. Meißner, Phys. Rev. Lett. 104 (2010) 142501.

[42] E. Epelbaum, H. Krebs, D. Lee, U.-G. Meißner, Eur. Phys. J. A45 (2010) 335.

[43] E. Epelbaum, H. Krebs, D. Lee, U.-G. Meißner, Phys. Rev. Lett. 106 (2011) 192501. 